\title{
Mild Encephalopathy with a Reversible Splenial lesion, a differenzial diagnosis to consider in children
}

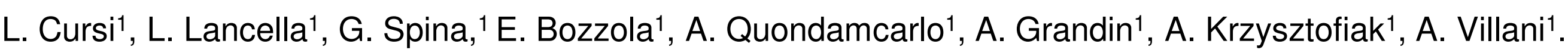

${ }^{1}$ Bambino Gesù Children Hospital, Pediatric and Infectious Disease Unit, Rome, Italy.

Background

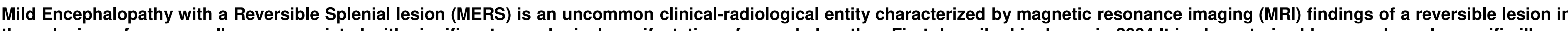

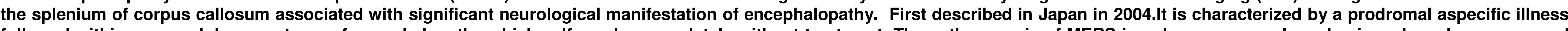

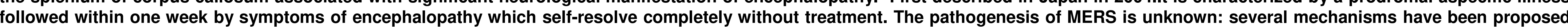
but the infectious trigger seems to be the most likely one. We report three cases of children with different clinical and radiological presentation, associated to an infection.

\section{Case Presentation Summary}

The first case is a 14 years old girl admitted for malaise, fever, headache and later a worsening of neurological aspect with confusion, psychomotor crisis, urinary and fecal retention. Laboratory tests showed hyponatremia and HHV6 infection. The instrumental exams (head Computed Tomography -TC- and MRI) conducted 4 days after the onset of the symptoms showed no pathological results. Analysis of cerebrospinal fluid (CSF): an increase in protein. Slow frontotemporal left waves were described to EEG. Alterations were highlighted to somatosensory evoked potentials of lower limbs. A new brain MRI was carried out 12 days after onset of symptoms with evidence of lesions in the corpus callosum at the splenius level, not present at the previous images and having characteristics compatible with MERS. Steroid therapy was started and a progressive resolution of the clinical condition and the normalization of laboratory and instrumental examinations were observed within one month of the onset of the symptoms.

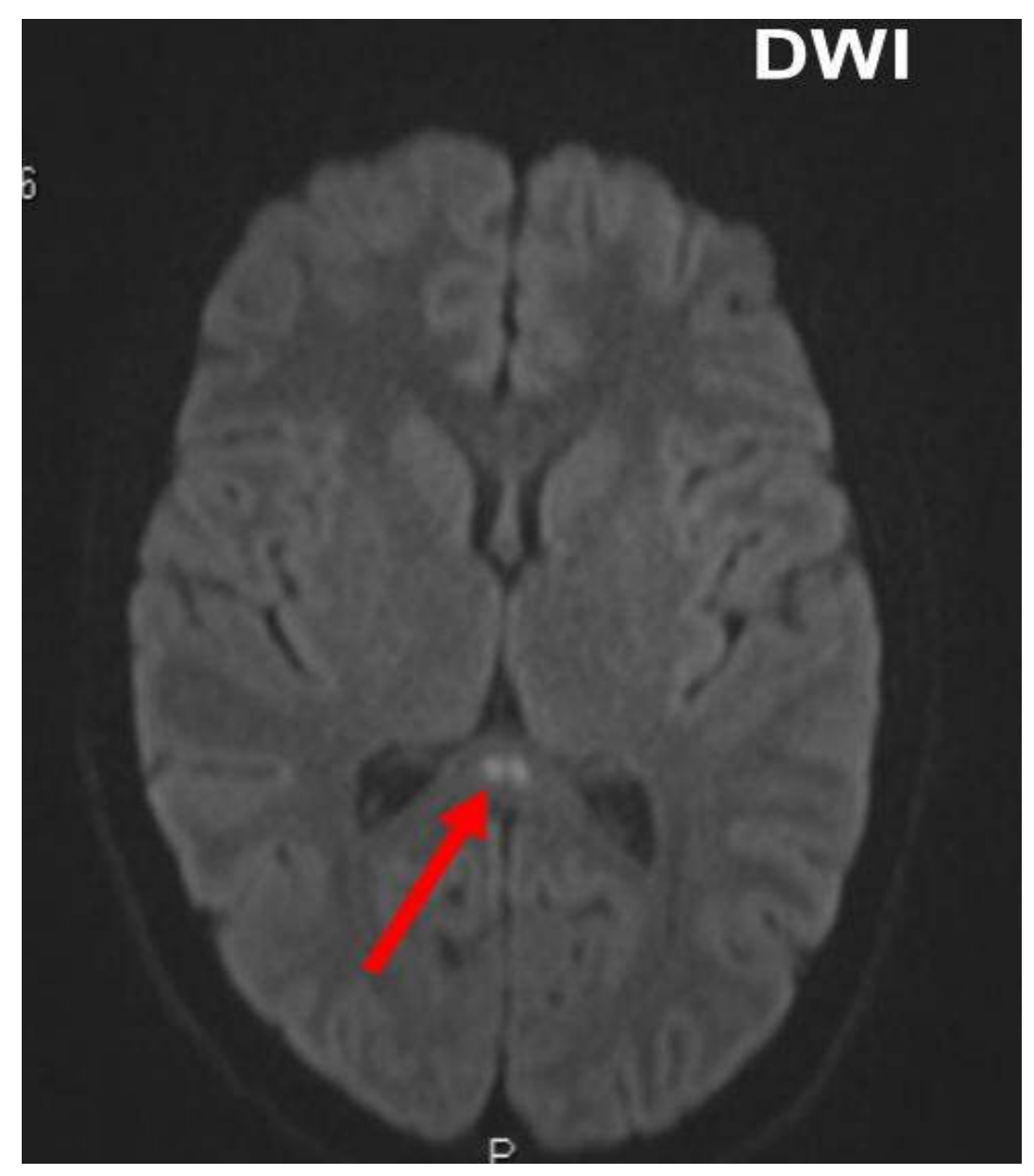

The symmetrical lesions showed fluid-attenuated inversion recovery images in diffusion-weighted images (DWI), w
corresponding diffusion restriction and no contrast enhancement.
The second patient is a 3 years old boy admitted for fever, hyponatremia and hypoglicemia. Neurological examination showed poor mental status, lethargy with absence of lower limb tendon reflexes. Cranial MRI showed a focal lesion in the splenium of the corpus callosum and in subcortical brain regions. The EEG revealed diffuse slow waves. Pharyngeal swab PCR was positive for Influenza B. A progressive resolution of the clinical condition and the normalization of laboratory and instrumental examinations were observed within two month of the onset of the symptoms after steroid treatment.

The third case is a 11 years old female admitted to our department because of fever, strabismus of the left eye and diplopia. Neuro-ophtalmologic evaluation was normal. Cranial MRI revealed the presence of high signal lesion in the splenium of corpus callosum. The laboratory tests showed HHV6 infections. A total clinical resolution was observed without any treatment.
Learning Points/Discussion

The evaluation of clinical manifestations of MERS is necessary to suspect the disease but it is not enough: the brain MRI conducted during the acute episode is mandatory for diagnosis. ${ }^{1}$ The onset of MERS is characterized by prodromal nonspecific symptoms as fever, cough, vomiting or diarrhea followed after few days and within one week by headache, altered consciousness, drowsiness, seizures, ataxia, vertigo, delirium and behavioural change. ${ }^{3}$ The symptoms subside completely within a month without any sequelae. ${ }^{1,2}$ The brain MRI classically reveals lesions in the splenium of the corpus callosum (MERS type 1 lesions), as in our first and third patient, even if sometimes the lesions extend to other areas of the corpus callosum and to adjacent parenchymal white matter (MERS type 2 lesions), as in second patient ${ }^{4}$. The localization of the disease in the brain justifies the clinical manifestations as disorder of motor control, spatial orientation, vision, hearing, and language-related behaviors: indeed, the corpus callosum is a fiber bundle that projects into prefrontal, premotor, primary motor, primary sensory, parietal, temporal, and occipital cortical areas. The pathogenesis of MERS is still not fully understood. A probable role is due to intra-myelinic edema, ${ }^{1}$ interstitial edema in tightly packed fibers, or inflammatory infiltrate..$^{1,3}$ Such edema may develop either as a result of electrolyte/water imbalance, especially hypotonic hyponatremia, or axonal damage and oxidative stress due to myelin neurotoxins released by pathogens, ${ }^{1}$, mainly viruses - In our cases both hyponatremic and infectious mechanisms were present. The differentia diagnosis of MERS includes infections, ischemia, multiple sclerosis, lymphoma, acute disseminate encephalomyelitis, and posterior reversible encephalopathy. Methylprednisolone pulse therapy and IVIG are recommended for patients with infectious encephalopathy regardless of pathogen or clinic-radiological syndromes but there are no guidelines about the treatment of MERS. A review of literature reports patients with MERS with full recovery irrespective of the therapies, suggesting that there is no supporting evidence of treatments with steroids or IVIG for MERS so far.

\section{Conclusion}

MERS is an important differential diagnosis to consider in children presenting with acute encephalopathy/encephalitis. MERS in clinical practice is very low, but knowledges about its existence are essential to suspect and manage it. 